\title{
Article
}

\section{Surface EMG signal normalisation and filtering improves sensitivity of equine gait analysis}

St George, Lindsay Blair, Roy, SH, Richards, James, Sinclair, Jonathan Kenneth and Hobbs, Sarah Jane

Available at https://clok.uclan.ac.uk/28496/

St George, Lindsay Blair orcid iconORCID: 0000-0002-5531-1207, Roy, SH, Richards, James orcid iconORCID: 0000-0002-4004-3115, Sinclair, Jonathan Kenneth orcid iconORCID: 0000-0002-2231-3732 and Hobbs, Sarah Jane orcid iconORCID: 0000-0002-1552-8647 (2019) Surface EMG signal normalisation and filtering improves sensitivity of equine gait analysis. Equine and Comparative Exercise Physiology, 15 (3). pp. 173-185. ISSN 1478-0615

It is advisable to refer to the publisher's version if you intend to cite from the work. http://dx.doi.org/10.3920/CEP190028

For more information about UCLan's research in this area go to

http://www.uclan.ac.uk/researchgroups/ and search for <name of research Group>.

For information about Research generally at UCLan please go to http://www.uclan.ac.uk/research/

All outputs in CLoK are protected by Intellectual Property Rights law, including Copyright law. Copyright, IPR and Moral Rights for the works on this site are retained by the individual authors and/or other copyright owners. Terms and conditions for use of this material are defined in the policies page. 


\title{
Surface EMG signal normalisation and filtering improves sensitivity of equine gait analysis
}

St. George, L. ${ }^{1}$, Roy S.H. ${ }^{3}$, Richards, J. ${ }^{2}$, Sinclair, J. ${ }^{1}$, Hobbs, S. J ${ }^{1}$.

${ }^{1}$ University of Central Lancashire, Centre for Applied Sport and Exercise Sciences, Preston, Lancashire PR1 2HE, United Kingdom;

${ }^{2}$ University of Central Lancashire, Allied Health Research Unit, Preston, Lancashire PR1 2HE, United Kingdom;

${ }^{3}$ Delsys Inc., Natick, MA 01760

Corresponding author: Dr. Lindsay St. George,

University of Central Lancashire,

Preston, PR1 2HE, United Kingdom.

Tel: 01772893775 Email: lbstgeorge@uclan.ac.uk

Running Header: Effect of normalisation and filtering on equine sEMG outcome measures.

\begin{abstract}
Low-frequency noise attenuation and normalisation are fundamental signal processing (SP) methods for surface electromyography (sEMG), but are absent, or not consistently applied, in equine biomechanics. The purpose of this study was to examine the effect of different bandpass filtering and normalisation conventions on sensitivity for identifying differences in sEMG amplitude-related measures, calculated from leading $(\mathrm{LdH})$ and trailing hindlimb $(\mathrm{TrH})$ during canter, where between-limb differences in vertical loading are known. sEMG and 3Dkinematic data were collected from the right Biceps Femoris in 10 horses during both canter leads. Peak hip and stifle joint angle and angular velocity were calculated during stance to verify between-limb biomechanical differences. Four SP methods, with and without normalisation and high-pass filtering, were applied to raw sEMG data. Methods 1 (M1) to 4 (M4) included DC-offset removal and full-wave rectification. Method 2 (M2) included additional normalisation relative to maximum sEMG across all strides. Method 3 (M3) included additional high-pass filtering (Butterworth $4^{\text {th }}$ order, $40 \mathrm{~Hz}$ cut-off), for artefact attenuation. M4 included the addition of high-pass filtering and normalisation. Integrated EMG (iEMG) and average rectified value (ARV) were calculated using processed sEMG data from M1 - M4, with stride duration as the temporal domain. sEMG parameters, within M1 - M4, and kinematic parameters were grouped by $\mathrm{LdH}$ and $\mathrm{TrH}$ and compared using repeated measures ANOVA. Significant between-limb differences for hip and stifle joint kinematics were found, indicating functional differences in hindlimb movement. M2 and M4, revealed significantly greater iEMG and ARV for $\mathrm{LdH}$ than $\operatorname{TrH}(\mathrm{p}<0.01)$, with M4 producing the lowest $p$ values and largest effects sizes. Significant between-limb differences in sEMG parameters were not observed with M1 and M3. The results indicate that equine sEMG SP should include normalisation and high-pass filtering to improve sensitivity for identifying differences in muscle function associated with biomechanical changes during equine gait.
\end{abstract}

Keywords: surface electromyography, horse, signal processing, high-pass filter, normalise

Conflict of Interest: none 


\section{Introduction}

Surface electromyography (sEMG) has long been used as a non-invasive tool for investigating the human neuromuscular system. Within the equine biomechanics field, the use of sEMG is relatively scarce compared to human studies but has gained popularity in the past 10 years. sEMG has proven to be a useful method for understanding equine muscle function during normal locomotion (Harrison et al., 2012; Jansen et al., 1992; Robert et al., 1999; Zsoldos et al., 2010a; Zsoldos et al., 2010b), but also for differentiating the biomechanical effects of speed (Robert et al., 2001a; Robert et al., 2002), incline (Crook et al., 2010; Hodson-Tole, 2006; Robert et al., 2000; Robert et al., 2001b), fatigue (Cheung et al., 1998; Colborne et al., 2001; Williams et al., 2013) and lameness (Zaneb et al., 2009) on equine gait. The relative ease of sEMG signal acquisition makes it an attractive tool for both human and equine researchers, but sEMG signal quality and processing techniques must be carefully considered for accurate analysis and interpretation of muscle function in response to changes in biomechanics.

Some of the factors that influence signal quality can be mitigated by technological advances in sensor design and complying with best practice for locating and adhering the sensor to the skin (Clancy et al., 2002; De Luca, 1997; De Luca et al., 2010; Roy et al., 2007). There are applications however when contamination of the low-frequency sEMG spectra, from baseline and movement artefact noise, and intra/ inter-individual subject characteristics, such as subcutaneous fat thickness, are unavoidable and of particular concern when interpreting the sEMG signal (De Luca et al., 2010; Halaki and Ginn, 2012; Kuiken et al., 2003; Lehman and McGill, 1999; Nordander et al., 2003). Methodological guidelines for human sEMG recording and processing have been published and describe optimal signal processing (SP) methods to mitigate these sources of error. Of these, the International Society of Electrophysiology and Kinesiology (ISEK) (Winter et al., 1980) and Standards for Reporting EMG data (Merletti and Di Torino, 1999), recommend SP methods that include high-pass filtering, for attenuating lowfrequency noise contamination (De Luca et al., 2010; Van Boxtel, 2001; Van Boxtel et al., 1998), and normalisation, for reducing inter and intra-subject variability (Burden, 2010; Halaki and Ginn, 2012; Lehman and McGill, 1999).

Unfortunately, standards for sEMG signal detection and processing in equine subjects are not currently available and methodological variation within equine sEMG literature is particularly evident for SP methods (Valentin and Zsoldos, 2016). Furthermore, fundamental lowfrequency noise attenuation and normalisation techniques are absent, or not consistently applied in the equine sEMG literature (Valentin and Zsoldos, 2016). Reliance on human subject-based sEMG guidelines for equine subjects is not recommended, as differences in size, mass, bipedal vs. quadrupedal gait and skin properties alone are sufficient to question their equivalence. Thus, the need for a best practice framework that follows human sEMG guidelines while taking into consideration the unique challenges associated with detecting and processing sEMG data from equine subjects, has been initiated (St. George et al., 2018; Valentin and Zsoldos, 2016). Our recent work (St. George et al. 2018) demonstrated that simply adopting human sEMG guidelines for removal of motion artefact for equine gait studies is not adequate. The removal of low-frequency noise contamination within sEMG signals obtained from Biceps Femoris and Triceps Brachii during trot and canter was found to be more effective using a high-pass filter with a $30-40 \mathrm{~Hz}$ cut-off frequency, when compared to the standard recommendation of a $10-20 \mathrm{~Hz}$ cut-off frequency shown for human studies (De Luca et al., 2010). Although the need for an equine-specific high-pass filtering cut-off has been 
demonstrated for optimal attenuation of signal noise in these studies, the practical effect of different high-pass filtering cut-offs on the sensitivity of sEMG outcome measures for equine gait analysis has not yet been investigated. Similar questions arise for SP practices involving sEMG signal normalisation. Valentin and Zsoldos (2016) reported that normalisation techniques are frequently absent in the equine sEMG literature, but no studies have demonstrated the consequences of this on the interpretation of equine sEMG data. In the human literature, Lehman and McGill (1999) investigated the effect of normalisation on the sensitivity of sEMG outcome measures for analysing the relationship between upper and lower rectus abdominus (RA) during a trunk curl exercise. When data were normalised to a maximum voluntary contraction (MVC) activity of the upper and lower RA were comparable, which was considered a clinically correct interpretation of muscle function, but when normalisation was omitted, a large asymmetry between upper and lower RA activity was observed (Lehman and McGill, 1999). High-pass filtering and normalisation have therefore been shown to improve the sensitivity of accurately interpreting human sEMG signal findings in relation to changes in biomechanics. However, no studies to date have demonstrated their effect on sensitivity for identifying differences in equine muscle function during gait analysis.

The purpose of this study is to test whether adopting a more rigorous SP protocol for sEMG filtering and normalisation provides greater sensitivity, reflected by smaller P-values and larger effect sizes, in identifying differences in muscle activation during equine gait, when compared to the current standard. For this example, sEMG and three-dimensional kinematic data, obtained unilaterally from right hindlimb and the vertebral head of the right Biceps Femoris (BF) during canter, were chosen a priori. The canter is a three-phase asymmetrical gait with a footfall pattern as follows: 1) trailing hindlimb ( $\mathrm{TrH})$; 2) leading hindlimb ( $\mathrm{LdH})$ and trailing forelimb (TrF) (as a diagonal pair); and 3) leading forelimb (LdF). During canter, biomechanically different demands are placed on hindlimb, depending on these phases of gait, with the LdH experiencing greater vertical loading (Merkens et al., 1993) and flexion of stifle and tarsal joints (Back et al., 1997) during stance than TrH. Because the BF acts to adduct the hindlimb and extend the hip and stifle joints during stance (Payne et al., 2005; Robert et al., 1999), the differences in loading are expected to produce different levels of BF muscle activation, which are measured as differences in sEMG signal amplitudes during equine gait analysis. In this study, peak joint angle and angular velocity are calculated for the hip and stifle joint during the stance phase to characterize biomechanical differences for the equine subjects during the periods of sEMG signal measurement of the BF muscle. To evaluate the effects of band-pass filtering and normalisation, four different SP methods were applied to the BF sEMG signals. Methods 1 (M1) to 4 (M4) included DC-offset removal and full-wave rectification. Method 2 (M2) included additional normalisation relative to maximum sEMG across all strides. Method 3 (M3) included additional high-pass filtering (Butterworth $4^{\text {th }}$ order, $40 \mathrm{~Hz}$ cut-off), for artefact attenuation and M4 included the addition of both high-pass filtering and normalisation. Commonly employed amplitude-based sEMG parameters were computed from the processed sEMG signals from each method to quantify the magnitude of muscle activation. It is hypothesised that incorporating normalisation with the most recent recommendations for equine filtering at $40 \mathrm{~Hz}$ (St. George et al., 2018) will provide the greatest sensitivity for identifying statistically significant differences in BF sEMG activation between $\mathrm{LdH}$ and $\mathrm{TrH}$, which correspond to between-hindlimb differences in joint kinematics during canter. 


\section{Methods}

Ethical approval for this study was obtained from the University of Central Lancashire's Animal Projects Committee (RE/13/04/SH). Written informed consent was obtained from all horse owners, riders and handlers prior to data collection.

Data were collected from 10 horses (age: $9.7 \pm 2.6$ years, height: $161.9 \pm 6.3 \mathrm{~cm}$, sex: 7 geldings, 3 mares, breed: various). All horses were in training and free from lameness, as defined by their owner. sEMG and 3D kinematic data were collected unilaterally from the right hindlimb at $2088 \mathrm{~Hz}$ and $232 \mathrm{~Hz}$ respectively during ridden canter trials. Unilateral sEMG and kinematic data were collected during right and left canter lead trials, when the right hindlimb functioned as $\mathrm{LdH}$ and $\mathrm{TrH}$, respectively. This was done to study how the different SP methods influence measures of muscle activation from one muscle when it is analysed under different loading conditions. sEMG data were collected from right BF using wireless sEMG sensors (Trigno $^{\mathrm{TM}}$, Delsys Inc., USA), with a bi-polar parallel bar electrode configuration and an interelectrode distance of $10 \mathrm{~mm}$. Sensor sites for BF were approximately halfway between the third trochanter and patella, and approximately $9 \mathrm{~cm}$ cephalad to the cranial margin of Semitendinosus (Schuurman et al., 2003). Prior to sensor adhesion, sensor sites were prepared by removing all hair and thoroughly cleaning with isopropyl alcohol wipes. A small amount of saline solution was applied to the electrode bars to act as an electrolytic solution (Clancy et al., 2002; Cram and Rommen, 1989). Sensors were then adhered to prepared sites using a combination of Delsys Adhesive Surface Interface strips (Delsys Inc., USA) and strips of double-sided tape, which were applied to the top and bottom of the sensor above each electrode pair. The sensor was positioned on the muscle belly, with electrode bars oriented perpendicular to the underlying muscle fibre direction (De Luca, 1997; Hermens et al., 2000).

Three-dimensional kinematic data were collected to detect right hindlimb hoof impact and liftoff gait events for stride segmentation. In addition, kinematic data were collected to calculate peak joint angle and angular velocity for the hip and stifle joints during stance phase, for which the BF functions as an extensor (Payne et al., 2005; Robert et al., 1999), as a means of analysing muscle activity in relation to expected biomechanical differences in hindlimb function. Spherical retro reflective markers, (25 mm diameter) (Qualisys AB, Sweden) were positioned over the following anatomical landmarks on the right hindlimb: the most ventral part of the tuber coxae, greater trochanter, lateral epicondyle of the femur, talus and the center of rotation of the metatarsalphalangeal and distal interphalangeal joints. A marker was also attached over the croup for stride velocity calculation. Data were collected using eight Qualisys Oqus cameras (Qualisys AB, Sweden). Cameras were positioned side-by-side in a linear configuration and an extended calibration was conducted to collect data from multiple strides (Figure 1). The calibration volume was approximately $8 \mathrm{~m}$ in length.

Figure 1. Equipment set-up for data collection showing camera configuration and instrumented equine subject.

\subsection{Data Collection}

Data were collected during ridden canter trials using Qualisys Track Manager (QTM) software (Qualisys AB, Sweden). Five different riders, with similar experience and ability, rode the horses during data collection. Each horse was ridden by their usual rider, who either owned or had experience riding them. Kinematic and sEMG data were synchronously acquired using an 
external trigger system (Delsys Trigger Module, Delsys Inc., USA). A static trial was initially recorded for each horse. Following the static trial, each horse progressed through the capture volume during ridden canter. Horses were permitted to travel at their preferred velocity and riders were instructed to position horses adjacent to placing poles, positioned on the ground approximately $4.5 \mathrm{~m}$ from the cameras to demarcate the optimal capture volume (Figure 1). Three successful trials were collected from each horse during right and left canter lead, which were randomised. A trial was successful when the horse held the canter and the correct canter lead through the calibrated volume and did not deviate from the optimal capture volume. The number of strides collected within the calibrated volume differed between horses, largely due to differences in sizes and stride lengths. Thus, the number of strides collected from each horse was not standardised in this study.

\subsection{Data Processing and Analysis}

Kinematic data were tracked in QTM and both kinematic and sEMG data were imported into Visual3D (C-Motion Inc., USA) for further analysis. Kinematic data were interpolated and low-pass filtered (Butterworth 4th order), with a cut-off frequency of $12 \mathrm{~Hz}$, as determined using residual analysis. Hindlimb hoof impact and lift-off events were calculated from kinematic data using a hindlimb sagittal plane angle in accordance with Holt et al. (2017). Kinematic gait events were applied to sEMG signals to segment the signal into stance and swing phases. A constant delay of $20 \mathrm{~ms}$ between kinematic and sEMG data was corrected for by shifting sEMG signals forward by 5 frames prior to applying kinematic gait events. To calculate stride velocity, the first derivative of the croup marker was calculated in the sagittal plane, and the average velocity was calculated between consecutive hoof impact events. Kinematic markers were used to define the distal and proximal ends of the pelvis, femur, tibia and third metatarsal segments of the right hindlimb. A segment coordinate system (SCS) was defined for segment, with the $\mathrm{X}$ axis as mediolateral, $\mathrm{Y}$ axis as cranio-caudal and $\mathrm{Z}$ axis as axial. Joint angles were calculated in the sagittal plane, as rotation around the SCS X axis, using the proximal and distal segments for each joint. Joint angular velocity was determined by calculating the first derivative of the hip and stifle joint angle signals. Flexion was defined as positive and extension as negative. During stance phase, vertical forces are primarily absorbed by shortening of the hindlimb between the stifle joint and hoof (Hjerten et al., 1994), while the distance between the stifle joint and tuber coxae increases as the hip joint undergoes extension (Back et al., 1996; Back et al., 1995; Hodson et al., 2001). Thus, peak joint angle and angular velocity were calculated for hip joint extension and stifle joint flexion during stance phase.

Raw sEMG signals were differentially amplified by a factor gain of 909 , a common-mode rejection ratio (CMRR) of $>80 \mathrm{~dB}$ and an internal Butterworth high-pass $(20 \pm 5 \mathrm{~Hz}$ cut-off, > $40 \mathrm{~dB} / \mathrm{dec}$ ) and low-pass filter $(450 \pm 50 \mathrm{~Hz}$ cut-off, $>80 \mathrm{~dB} / \mathrm{dec})$. Post-processing of signals was conducted in Visual3D, where four SP methods were applied to the raw SEMG data. M1 represents the most commonly applied SP method within existing equine sEMG literature and includes DC-offset removal and full-wave rectification of signals following acquisition. M2 follows the same protocol as M1 but includes additional normalisation relative to a maximal reference voluntary contraction (RVC) (Lehman and McGill, 1999; Sousa and Tavares, 2012; Yang and Winter, 1984). In this instance, the RVC represents the maximum sEMG outcome measure observed across all canter strides within each horse. The use of an RVC is based on recommendations from human studies where obtaining a maximal voluntary contraction 
(MVC) is not possible or difficult to obtain, for example in participants experiencing pain or with neurologic disorders (Burden and Bartlett, 1999; Dankaerts et al., 2004; Lehman and McGill, 1999; Yang and Winter, 1984). M3 and M4 follow the same SP protocol as M1 but include additional high-pass filtering using a Butterworth 4th order filter with a $40 \mathrm{~Hz}$ cut-off frequency based on recent equine recommendations (St. George et al., 2018), with M4 including both high-pass filtering and normalisation relative to the RVC, which have not been adopted routinely in equine studies.

Integrated EMG (iEMG) and average rectified value (ARV) represent commonly reported amplitude-based outcome measures in equine sEMG literature for studies examining differences in muscle function during gait (Robert et al., 2001a; Robert et al., 2001b; Robert et al., 2000; Robert et al., 2002; Zaneb et al., 2009; Zsoldos et al., 2010a; Zsoldos et al., 2010b). iEMG represents the area under the voltage curve, where the sEMG signal is integrated over a specified time interval, and ARV represents the mean value of the full-wave rectified sEMG signal over a specified time interval (Merletti and Di Torino, 1999; Winter et al., 1980). The effect of the different SP methods was therefore evaluated using iEMG and ARV, which were calculated in accordance with Merletti and Di Torino (1999) and Winter et al. (1980) using the full-wave rectified signal from Methods 1 - 4 and stride duration as the time interval.

\subsection{Statistical analysis}

For each sEMG outcome measure (iEMG, ARV), data from LdH and TrH were grouped within each SP method (M1, M2, M3, M4). Ensemble averages (mean \pm SD) were calculated for each sEMG (iEMG and ARV) and kinematic (peak joint angle and peak joint angular velocity) outcome measure to examine differences between limbs. One-way repeated measures ANOVAs were used to compare kinematic and sEMG outcome measures from LdH and $\mathrm{TrH}$, with sEMG outcome measures compared within each SP method. Significant differences were identified at $\mathrm{P}<0.05$ and effect sizes were established using partial eta ${ }^{2}\left(\mathrm{pn}^{2}\right)$. Sensitivity for identifying differences were therefore based on conditions which provided the lowest $p$-value and largest effect size.

\section{Results}

Across all horses, 115 strides were analysed, with 62 and 53 strides analysed when the right hindlimb acted as $\operatorname{TrH}$ and $\mathrm{LdH}$, respectively. Stride velocity was $4.6 \pm 0.4 \mathrm{~m} / \mathrm{s}$ across all horses. Mean peak joint angle and angular velocity $( \pm \mathrm{SD})$ data for the stifle and hip joints are presented in Figure 2 and Table 1 for normalized canter strides. During stance phase, the LdH exhibited significantly greater stifle joint flexion $(p=0.001)$ and hip joint extension $(p=0.000)$ than the TrH. Significantly greater peak flexion velocity was observed in the LdH for the stifle joint $(\mathrm{p}=0.000)$ and significantly greater peak extension velocity was observed in the $\mathrm{TrH}$ for the hip joint $(\mathrm{p}=0.037)$ during stance phase.

Figure 2. Mean (bold line) and standard deviation (shaded area) joint angle $\left(^{\circ}\right.$ ) and joint angular velocity data ( $\%$ s) for a) hip joint angle, b) stifle joint angle, c) hip joint angular velocity, d) stifle joint angular velocity from $\mathrm{LdH}$ (blue) and $\mathrm{TrH}$ (red). Data are normalised over one canter stride, with the hoof-lift off event demarcated by the green vertical line. Flexion was defined as positive and extension as negative. Overall average peak joint angle and peak joint angular velocity events are presented on corresponding graphs as red and blue arrows for $\mathrm{TrH}$ and $\mathrm{LdH}$, respectively. 
Table 1. Mean ( \pm sd) peak joint angle $\left({ }^{\circ}\right)$ and peak joint angular velocity data $(\% / s)$ from the stifle joint and hip joint during stance phase. Data are grouped according to limb (LdH and $\mathrm{TrH})$. Differences between $\mathrm{LdH}$ and $\mathrm{TrH}$ are presented for each joint as $\mathrm{P}$ values and effects sizes $\left(\mathrm{pn}^{2}\right)$. Significant differences $(\mathrm{P}<0.05)$ between limbs are denoted by bold text.

\begin{tabular}{|l|l|l|l|l|}
\hline & TrH & LdH & P value & $\mathrm{pn}^{2}$ \\
\hline Hip Joint Angle $\left(^{\circ}\right)$ & $-33.7(8.7)$ & $-44.1(7.6)$ & $\mathbf{0 . 0 0 0}$ & 0.823 \\
\hline Stifle Joint Angle $\left(^{\circ}\right)$ & $33.9(6.3)$ & $39.3(5.5)$ & $\mathbf{0 . 0 0 1}$ & 0.723 \\
\left.\hline${\text { Hip Joint Angular Velocity }\left({ }^{\circ} / \mathrm{s}\right)}\right)$ & $-226.6(40.8)$ & $-181.4(37.9)$ & $\mathbf{0 . 0 3 7}$ & 0.399 \\
\hline Stifle Joint Angular Velocity $(\%)$ & $342.6(97.3)$ & $481.5(81.4)$ & $\mathbf{0 . 0 0 0}$ & 0.866 \\
\hline
\end{tabular}

Descriptive and inferential statistics for sEMG outcome measures are presented in Table 2 and 3 and show that LdH exhibited greater mean ARV and iEMG values than TrH across all SP methods. Between limb differences for ARV and iEMG were only significant when normalisation was applied in M2 and M4, with the addition of high-pass filtering in M4 resulting in a lower $p$ value and higher effect size (ARV: $p=0.002$, iEMG: $p=0.002$ ) than M2 (ARV: $\mathrm{p}=0.017$, iEMG: $\mathrm{p}=0.016$ ). $\mathrm{M} 1$ and M3, which did not include normalisation, did not detect significant differences between limbs for iEMG and ARV ( $p>0.05)$. For both iEMG and $\mathrm{ARV}$, the addition of high-pass filtering in M3 was again found to produce higher effect sizes and lower $p$ values that approached significance (ARV: $p=0.066$, iEMG: $p=0.074$ ) than Method 1 (ARV: $p=0.101$, iEMG: $\mathrm{p}=0.109$ ). Combined mean and standard deviation data from all subjects in Tables 2 and 3 reveal that normalisation, employed in M2 and M4, resulted in reduced standard deviation for iEMG and ARV outcome measures. Figures 3 and 4 illustrate this finding by showing decreased intrasubject variability and more distinct between-limb differences when normalisation is applied to ARV data from two different horses (Figure 3 and $4 \mathrm{c}, \mathrm{d})$. Figures 3 and 4 also illustrate the effect of high-pass filtering on between-limb differences, which are in accordance with findings presented in Tables 2 and 3. When highpass filtering is applied to sEMG signals in Figures 3 and 4 (b, d), which represent M3 and M4, respectively, a distinct between-limb difference is observed, with the LdH clearly showing greater amplitude of sEMG activity than TrH. In comparison, when high-pass filtering is not applied in Figures 3 and 4 (a, c), which represent M1 and M2, respectively, sEMG signals from $\mathrm{TrH}$ often overlap with signals from LdH. Thus, the omission of high-pass filtering in Figures 3 and 4 does not result in distinct between limb differences.

Table 2. Mean ( $\pm \mathrm{sd}$ ) for $\mathrm{iEMG}$, calculated using processed sEMG signals from Methods 1 to 4 and grouped according to limb ( $\mathrm{LdH}$ and $\mathrm{TrH})$. Differences between $\mathrm{LdH}$ and $\mathrm{TrH}$ within Methods 1 to 4 are presented for each outcome measure as $\mathrm{P}$ values and effects sizes $\left(\mathrm{pn}^{2}\right)$. Significant differences $(\mathrm{P}<0.05)$ between limbs are denoted by bold text.

\begin{tabular}{|c|c|c|c|c|c|}
\hline $\begin{array}{l}\text { Outcome } \\
\text { Measure }\end{array}$ & Signal Processing Method & TrH & LdH & $\begin{array}{l}P \\
\text { value }\end{array}$ & $\mathbf{p n}^{2}$ \\
\hline iEMG & $\begin{array}{l}\text { Method } 1 \\
\text { - } \quad \text { DC offset removal } \\
\text { - } 20-450 \mathrm{~Hz} \text { band pass filtered } \\
(\mu \mathrm{V} . \mathrm{s})\end{array}$ & $\begin{array}{l}18.2 \\
(13.7)\end{array}$ & $\begin{array}{l}28.6 \\
(30.8)\end{array}$ & 0.109 & 0.288 \\
\hline
\end{tabular}




\begin{tabular}{|c|c|c|c|c|}
\hline $\begin{array}{l}\text { Method } 2 \\
\text { - } \quad \text { DC offset removal } \\
\text { - } 20-450 \mathrm{~Hz} \text { band pass filtered } \\
\text { - } \quad \text { Normalised } \\
\text { (\% Maximum Value) }\end{array}$ & $\begin{array}{l}65.1 \\
(17.2)\end{array}$ & $\begin{array}{l}82.1 \\
(7.1)\end{array}$ & 0.016 & 0.536 \\
\hline $\begin{array}{l}\text { Method } 3 \\
\text { - } \quad \text { DC offset removal } \\
\text { - } 20-450 \mathrm{~Hz} \text { band pass filtered } \\
\text { - } \quad \text { Butterworth high-pass filtered }(40 \mathrm{~Hz} \\
\text { cut-off }) \\
(\mu \mathrm{V} . \mathrm{s})\end{array}$ & $\begin{array}{l}10.4 \\
(6.6)\end{array}$ & $\begin{array}{l}17.7 \\
(17.2)\end{array}$ & 0.074 & 0.345 \\
\hline $\begin{array}{l}\text { Method } 4 \\
\text { - } \quad \text { DC offset removal } \\
\text { - } 20-450 \mathrm{~Hz} \text { band pass filtered } \\
\text { - } \quad \text { Butterworth high-pass filtered }(40 \mathrm{~Hz} \\
\quad \text { cut-off) } \\
\text { - Normalised } \\
\text { (\% Maximum Value) }\end{array}$ & $\begin{array}{l}57.4 \\
(17.0)\end{array}$ & $\begin{array}{l}79.1 \\
(6.8)\end{array}$ & 0.002 & 0.720 \\
\hline
\end{tabular}

Table 3. Mean ( \pm sd) for ARV, calculated using processed sEMG signals from Methods 1 to 4 and grouped according to limb ( $\mathrm{LdH}$ and $\mathrm{TrH})$. Differences between $\mathrm{LdH}$ and $\mathrm{TrH}$ within Methods 1 to 4 are presented for each outcome measure as $\mathrm{P}$ values and effects sizes $\left(\mathrm{pn}^{2}\right)$. Significant differences $(\mathrm{P}<0.05)$ between limbs are denoted by bold text.

\begin{tabular}{|c|c|c|c|c|c|}
\hline $\begin{array}{l}\text { Outcome } \\
\text { Measure }\end{array}$ & Signal Processing Method & TrH & LdH & $\begin{array}{l}P \\
\text { value }\end{array}$ & $\mathbf{p n}^{2}$ \\
\hline \multirow[t]{4}{*}{ ARV } & $\begin{array}{l}\text { Method } 1 \\
\text { - } \quad \text { DC offset removal } \\
\text { - } 20-450 \mathrm{~Hz} \text { band pass filtered } \\
(\mu \mathrm{V})\end{array}$ & $\begin{array}{l}30.6 \\
(22.1)\end{array}$ & $\begin{array}{l}47.8 \\
(49.4)\end{array}$ & 0.101 & 0.300 \\
\hline & $\begin{array}{l}\text { Method } 2 \\
\text { - } \quad \text { DC offset removal } \\
\text { - } 20-450 \mathrm{~Hz} \text { band pass filtered } \\
\text { - } \quad \text { Normalised } \\
\text { (\% Maximum Value) }\end{array}$ & $\begin{array}{l}65.2 \\
(17.4)\end{array}$ & $\begin{array}{l}82.4 \\
(7.6)\end{array}$ & 0.017 & 0.533 \\
\hline & $\begin{array}{l}\text { Method } 3 \\
\text { - } \quad \text { DC offset removal } \\
\text { - } 20-450 \mathrm{~Hz} \text { band pass filtered } \\
\text { - } \quad \text { Butterworth high-pass filtered }(40 \mathrm{~Hz} \\
\quad \text { cut-off }) \\
(\mu \mathrm{V})\end{array}$ & $\begin{array}{l}14.1 \\
(12.6)\end{array}$ & $\begin{array}{l}24.9 \\
(30.1)\end{array}$ & 0.066 & 0.362 \\
\hline & $\begin{array}{l}\text { Method } 4 \\
\text { - } \quad \text { DC offset removal } \\
\text { - } 20-450 \mathrm{~Hz} \text { band pass filtered } \\
\text { - } \quad \text { Butterworth high-pass filtered }(40 \mathrm{~Hz} \\
\quad \text { cut-off) } \\
\text { - } \quad \text { Normalised }\end{array}$ & $\begin{array}{l}57.4 \\
(16.7)\end{array}$ & $\begin{array}{l}78.9 \\
(6.6)\end{array}$ & 0.002 & 0.710 \\
\hline
\end{tabular}


Figure 3. Mean (bold line) and standard deviation (shaded area) sEMG ARV from Horse 2, obtained from right Biceps Femoris during left lead (red signals) and right lead (blue signals) canter when the right hindlimb functions as $\mathrm{TrH}$ and $\mathrm{LdH}$, respectively. Data are normalised over one canter stride, with the green vertical line on the $\mathrm{x}$-axis representing the hoof-lift off event. sEMG signals are smoothed using an RMS filter (window length: $0.125 \mathrm{~s}$, window overlap: $0.121 \mathrm{~s}$ ). The different signal processing methods are represented by a) Method 1, b) Method 3, c) Method 2, d) Method 4.

Figure 4. Mean (bold line) and standard deviation (shaded area) sEMG ARV data from Horse 4, obtained from right Biceps Femoris during left lead (red signals) and right lead (blue signals) canter when the right hindlimb functions as $\mathrm{TrH}$ and $\mathrm{LdH}$, respectively. Data are normalised over one canter stride, with the green vertical line on the $\mathrm{x}$-axis representing the hoof-lift off event. sEMG signals are smoothed using an RMS filter (window length: $0.125 \mathrm{~s}$, window overlap: $0.121 \mathrm{~s}$ ). The different signal processing methods are represented by a) Method 1, b) Method 3, c) Method 2, d) Method 4.

Figure 5 provides an individual example of how the application of a Butterworth high-pass filter with a $40 \mathrm{~Hz}$ cut-off frequency, as applied in M3 and M4, can influence both ARV and iEMG outcome measures and the interpretation of between-limb differences for $\mathrm{LdH}$ and $\mathrm{TrH}$. A comparison of band-pass filtered sEMG signals from $\mathrm{TrH}$ and $\mathrm{LdH}$ in Figure 5 (a, b) and their corresponding full-wave rectified signals in Figure 5 (c, d) with high-pass filtered signals in Figure $5(\mathrm{~g}-\mathrm{j})$ illustrates how additional high-pass filtering alters the amplitude of sEMG activation by removing low-frequency artefacts. The influence of high-pass filtering on outcome measures are evidenced in Figure $5(\mathrm{c}, \mathrm{d})$, where failure to apply high-pass filtering results in TrH exhibiting greater ARV and iEMG values than LdH. In contrast, the application of high-pass filtering in Figure 5 (e, f) results in LdH showing greater ARV and iEMG values, which is in accordance with overall results from this study (Table 2 and 3) and previous biomechanical literature describing functional differences between $\mathrm{LdH}$ and $\mathrm{TrH}$ during canter. Thus, failure to high-pass filter sEMG signals can lead to erroneous interpretation of results. In accordance with Tables 2 and 3 and Figures 3 and 4, Figure 5 (k. 1) also provides a visual representation of how M4's combination of additional high-pass filtering, to attenuate lowfrequency noise sources, and normalisation, to reduce intra subject variability, results in the greatest difference between $\mathrm{LdH}$ and $\mathrm{TrH}$ for sEMG outcome measures.

Figure 5. sEMG data obtained from right Biceps Femoris of Horse 2 over one left lead (red signals) and one right lead (blue signals) canter stride when the right hindlimb functions as TrH and LdH, respectively. Signal processing steps for Methods $1-4$ are illustrated as follows: a, b) band-pass filtered signals $(20-450 \mathrm{~Hz}), \mathrm{c}, \mathrm{d})$ full-wave rectification of band-pass filtered signals in a. and b. (Method 1), e, f) normalisation of band-pass filtered and full-wave rectified signals in c. and d. using maximum observed value (Method 3), g, h) band-pass filtered (20 $450 \mathrm{~Hz}$ ) and high-pass filtered (40 Hz cut-off frequency) signals, $i, j$ ) full-wave rectification of band-pass and high-pass filtered signals in g. and h. (Method 2), k, l) normalisation of bandpass filtered, high-pass filtered and full-wave rectified signals in i. and j. using maximum observed value (Method 4). iEMG and ARV data are provided for corresponding signals. The 300 hoof-lift off event is represented by the green tick on the $\mathrm{x}$-axis. 


\section{Discussion}

In this study, sEMG data were obtained from the right BF during canter, to compare the sensitivity of four different SP methods for identifying differences in muscle activity that results from known differences in limb loading between $\mathrm{LdH}$ and $\mathrm{TrH}$. Although it is known from the literature that the $\mathrm{LdH}$ experiences the greatest peak vertical loading of approximately 1.2 times the horse's body weight, and the TrH experiences the smallest with peak vertical loading approximately equal to the horse's body weight (Merkens et al., 1993), this study provides further kinematic evidence for these functional differences within our data set. In this study, significantly greater stifle joint flexion was accompanied by significantly greater peak flexion velocity for $\mathrm{LdH}$ during stance, which is indicative of an increased rate of stifle joint loading than that observed in $\mathrm{TrH}$. Although significantly greater hip joint extension was found in the $\mathrm{LdH}$, this coincided with significantly lower peak hip joint extension velocity than $\mathrm{TrH}$, indicating that $\mathrm{TrH}$ experiences a greater rate of hip joint loading than $\mathrm{LdH}$. Previous equine EMG studies report BF activity from late swing phase to late stance phase during trot and canter and postulate that the BF functions eccentrically during stance phase to stabilise the hip and stifle joints during limb loading (Crook et al., 2010; Robert et al., 1999; Tokuriki and Aoki, 1995). Based on our findings from kinematic data, it is therefore argued that the BF generates eccentric muscle activity with a greater force in the $\mathrm{LdH}$ to stabilise the hip joint and prevent involuntary flexion of the stifle joint (Denoix, 2014; Robert et al., 1999), which experiences a greater joint loading rate than $\mathrm{TrH}$, during increased vertical limb loading (Merkens et al., 1993). Significantly higher BF muscle activity was observed in $\mathrm{LdH}$ than $\mathrm{TrH}$ when M2 and M4 were applied, which agrees with reported functional differences in $\mathrm{LdH}$ and $\mathrm{TrH}$ from the literature (Back et al., 1997; Merkens et al., 1993) and from kinematic data presented in this study. Thus, the significant increase in BF activity in the LdH, observed when M2 and M4 are applied, provides an accurate representation of BF activity during canter.

It is important to note that the SP methods employed in this study were not chosen arbitrarily. M1 was based on a review of existing equine sEMG literature and represents the most commonly employed sEMG SP method within this field. M4 was based on a combination of best practice for human sEMG SP, where the importance of low-frequency noise attenuation and normalisation techniques are well established (Burden, 2010; De Luca et al., 2010; Lehman and McGill, 1999) and recent, equine-specific recommendations for high-pass filtering (St. George et al., 2018). M2 and M3 provide intermediary SP methods, which were used to identify the individual contributions of normalisation and high-pass filtering for identifying differences in BF muscle activity between $\mathrm{LdH}$ and $\mathrm{TrH}$. Following the application of all SP methods, amplitude-based outcome measures were calculated and compared, revealing significant differences in muscle activity between $\mathrm{LdH}$ and $\mathrm{TrH}$ when M2 and M4 were applied, but that M1 and M3 did not provide a sensitive enough metric to detect significant differences. Thus, the hypothesis that following recommended guidelines for sEMG SP, which includes normalisation and high-pass filtering, enables the identification of functional differences in muscle activation that would otherwise be missed was accepted. Of all methods, M4 resulted in the greatest between-limb differences in muscle activity, as evidenced by the lowest $p$ values and highest effect sizes for iEMG and ARV. Thus, SP techniques used for M4 may serve as a basis for developing standardisation for equine sEMG SP. However, when considering why M4 produces outcome measures that best reflect biomechanical differences between hindlimbs at canter, as well as the highest magnitude of between-limb differences, it is important to 
discuss the relative contributions of combining the $40 \mathrm{~Hz}$ high-pass filtering with normalisation.

\subsection{Effect of high-pass filtering on outcome measures}

In human sEMG literature, movement artefact and baseline noise sources are known to contaminate the sEMG frequency spectra between 0 and $20 \mathrm{~Hz}$ (Clancy et al., 2002; De Luca et al., 2010; Van Boxtel, 2001). Such artefacts influence the shape of the sEMG frequency spectra and can dominate the total signal power, leading to erroneous interpretation of both spectral and amplitude-based sEMG signal outcome measures (De Luca et al., 2010; Van Boxtel, 2001). Thus, attenuation of low-frequency noise in human studies is achieved using appropriate high-pass filtering techniques, where a cut-off frequency $\geq 20 \mathrm{~Hz}$ is recommended for maximally attenuating artefacts whilst minimising the removal of true sEMG signal content (De Luca et al., 2010; Van Boxtel, 2001; Van Boxtel et al., 1998). A more recent study carried out a similar approach among horses, where a high-pass filter cut-off frequency of 30 to $40 \mathrm{~Hz}$ was recommended for sEMG signals obtained from the BF of equine subjects during canter (St. George et al., 2018). This recommended high pass filter was therefore employed for M3 and M4 in the current study.

The beneficial effects of low-frequency noise attenuation on decreased intrasubject variability and increased between-limb differences in muscle activity when M3 and M4 are applied are illustrated in Figures 3 - 4. Furthermore, evidence for potential misinterpretation of muscle activity when high-pass filtering is omitted from SP is presented in Figure 5 (c, d, e, f), where M1 and M2 produce greater iEMG and ARV for TrH than LdH. M3, which employed highpass filtering without normalisation, did not produce statistically significant differences in muscle activity between $\mathrm{LdH}$ and $\mathrm{TrH}$. However, in comparison to M1, which did not employ high-pass filtering, M3 produced greater between-limb differences with lower $\mathrm{p}$ values, lower standard deviation and higher effects sizes for iEMG and ARV. Statistical power depends on both sample size and effect size, thus with a higher effect size it is possible to detect significant differences with a smaller sample size (Sullivan and Feinn, 2012). In equine sEMG research, the ability to employ a smaller sample size is advantageous due to the challenges associated with data acquisition, for example behavioural constraints and the time-consuming skin preparation process. Therefore, although the addition of high-pass filtering in M3 did not detect statistically significant between-limb differences in BF activity, attenuating low-frequency noise sources improves the sensitivity of SP methods by decreasing inter and intrasubject variability and increasing the magnitude of between-limb differences in muscle activity, which can lead to decreased sample size requirements for equine sEMG studies.

\subsection{Effect of normalisation on outcome measures}

Normalisation converts the amplitude of an sEMG signal to a scaled value, generally the percentage of a MVC or RVC from a specific task (Burden, 2010; Lehman and McGill, 1999). This technique is fundamental for comparisons of amplitude-related sEMG outcome measures across subjects, muscles and trials/ days (Burden, 2010; Halaki and Ginn, 2012; Lehman and McGill, 1999; Mathiassen et al., 1995) due to sources of variability associated with relative differences in sensor location, among other factors (De Luca, 1997). However, this is the first known study to demonstrate the effect of normalisation on sensitivity for identifying differences in muscle activity in relation to biomechanical differences in equine gait. The effect of normalisation on outcome measures in this study are clearly illustrated in Tables 2 and 3, 
where application of M2 and M4 resulted in significantly greater BF activity in LdH compared to TrH. Standard deviation values in Tables 2 and 3 also show that the omission of normalisation in $\mathrm{M} 1$ and $\mathrm{M} 3$ resulted in increased variation in $\mathrm{EEMG}$ and ARV variables, which will have influenced the non-significant results in the statistical analysis. Findings from this study indicate that reduced standard deviation from normalisation represents the major contribution to significant statistical findings and is therefore recommended for equine sEMG SP. However, the contribution of high-pass filtering should not be overlooked, as it is the combination of high-pass filtering and normalisation in M4 that provided the most sensitive SP method for detecting differences in BF activity in relation to biomechanical differences between $\mathrm{LdH}$ and $\mathrm{TrH}$ during canter.

\section{Conclusion}

sEMG signals, obtained from BF during canter, exhibited significantly different amplitudebased outcome measures between $\mathrm{LdH}$ and $\mathrm{TrH}$ when normalisation and recommended bandpass filtering techniques for equine sEMG signals (St. George et al., 2018) were applied. The changes in muscle function that were observed were consistent with underlying biomechanical differences in hindlimb loading during canter. However, between limb differences were not observed when high-pass filtering and normalisation were omitted from SP. Therefore, functional between-limb differences may be missed depending on the SP procedures employed for equine gait analysis. More specifically, findings from this study illustrate the importance of including both appropriate band-pass filtering and normalisation techniques to facilitate accurate interpretation of the equine sEMG signal. It is our intent that these findings may accelerate further best practice guidelines and standardisation efforts within the equine sEMG field to facilitate knowledge transfer via consistent methodology.

\section{References}

Back, W., Schamhardt, H. and Barneveld, A., 1996. Are kinematics of the walk related to the locomotion of a warmblood horse at the trot? Veterinary Quarterly 18: 79-84.

Back, W., Schamhardt, H. and Barneveld, A., 1997. Kinematic comparison of the leading and trailing fore-and hindlimbs at the canter. Equine Veterinary Journal 29: 80-83.

Back, W., Schamhardt, H., Savelberg, H., Van Den Bogert, A., Bruin, G., Hartman, W. and Barneveld, A., 1995. How the horse moves: 2. Significance of graphical representations of equine hind limb kinematics. Equine Veterinary Journal 27: 39-45.

Burden, A., 2010. How should we normalize electromyograms obtained from healthy participants? What we have learned from over 25 years of research. Journal of Electromyography and Kinesiology 20: 1023-1035.

Burden, A. and Bartlett, R., 1999. Normalisation of EMG amplitude: an evaluation and comparison of old and new methods. Medical Engineering \& Physics 21: 247-257.

Cheung, T., Warren, L., Lawrence, L. and Thompson, K., 1998. Electromyographic activity of the long digital extensor muscle in the exercising Thoroughbred horse. Equine Veterinary Journal 30: 251-255.

Clancy, E.A., Morin, E.L. and Merletti, R., 2002. Sampling, noise-reduction and amplitude estimation issues in surface electromyography. Journal of Electromyography and Kinesiology 12: 1-16.

Colborne, G., Birtles, D. and Cacchione, I., 2001. Electromyographic and kinematic indicators of fatigue in horses: a pilot study. Equine Veterinary Journal 33: 89-93.

Cram, J.R. and Rommen, D., 1989. Effects of skin preparation on data collected using an EMG muscle-scanning procedure. Biofeedback and Self-regulation 14: 75-82. 
Crook, T., Wilson, A. and Hodson-Tole, E., 2010. The effect of treadmill speed and gradient on equine hindlimb muscle activity. Equine Veterinary Journal 42: 412-416.

Dankaerts, W., O’Sullivan, P.B., Burnett, A.F., Straker, L.M. and Danneels, L.A., 2004. Reliability of EMG measurements for trunk muscles during maximal and sub-maximal voluntary isometric contractions in healthy controls and CLBP patients. Journal of Electromyography and Kinesiology 14: 333-342.

De Luca, C.J., 1997. The use of surface electromyography in biomechanics. Journal of Applied Biomechanics 13: 135-163.

De Luca, C.J., Gilmore, L.D., Kuznetsov, M. and Roy, S.H., 2010. Filtering the surface EMG signal: Movement artifact and baseline noise contamination. Journal of Biomechanics 43: $1573-1579$.

Denoix, J.-M., 2014. Biomechanics and physical training of the horse. CRC Press.

Halaki, M. and Ginn, K., 2012. Normalization of EMG signals: to normalize or not to normalize and what to normalize to?, Computational intelligence in electromyography analysis-a perspective on current applications and future challenges. InTech.

Harrison, S.M., Whitton, R.C., King, M., Haussler, K.K., Kawcak, C.E., Stover, S.M. and Pandy, M.G., 2012. Forelimb muscle activity during equine locomotion. Journal of Experimental Biology 215: 2980-2991.

Hermens, H.J., Freriks, B., Disselhorst-Klug, C. and Rau, G., 2000. Development of recommendations for SEMG sensors and sensor placement procedures. Journal of Electromyography and Kinesiology 10: 361-374.

Hjerten, G., Drevemo, S. and Eriksson, L.E., 1994. Shortening of the hind limb in the horse during the stance phase. Equine Veterinary Journal 26: 48-50.

Hodson-Tole, E., 2006. Effects of treadmill inclination and speed on forelimb muscle activity and kinematics in the horse. Equine and Comparative Exercise Physiology 3: 61-72.

Hodson, E., Clayton, H. and Lanovaz, J., 2001. The hindlimb in walking horses: 1. Kinematics and ground reaction forces. Equine Veterinary Journal 33: 38-43.

Jansen, M., Schamhardt, H. and Hartman, W., 1992. Quantitative analysis of computeraveraged electromyographic profiles of intrinsic limb muscles in ponies at the walk. American Journal of Veterinary Research 53: 2343-2349.

Kuiken, T.A., Lowery, M. and Stoykov, N., 2003. The effect of subcutaneous fat on myoelectric signal amplitude and cross-talk. Prosthetics and Orthotics International 27: 48-54.

Lehman, G.J. and McGill, S.M., 1999. The importance of normalization in the interpretation of surface electromyography: a proof of principle. Journal of Manipulative and Physiological Therapeutics 22: 444-446.

Mathiassen, S., Winkel, J. and Hägg, G., 1995. Normalization of surface EMG amplitude from the upper trapezius muscle in ergonomic studies-a review. Journal of Electromyography and Kinesiology 5: 197-226.

Merkens, H.W., Schamhardt, H.C. and Hartman, W., 1993. Ground reaction force patterns of Dutch Warmbloods at the canter. American journal of veterinary research 54: 670-674.

Merletti, R. and Di Torino, P., 1999. Standards for reporting EMG data. Journal of Electromyography and Kinesiology 9: 3-4.

Nordander, C., Willner, J., Hansson, G.-A., Larsson, B., Unge, J., Granquist, L. and Skerfving, S., 2003. Influence of the subcutaneous fat layer, as measured by ultrasound, skinfold calipers and BMI, on the EMG amplitude. European Journal of Applied Physiology 89: 514-519.

Payne, R., Hutchinson, J., Robilliard, J., Smith, N. and Wilson, A., 2005. Functional specialisation of pelvic limb anatomy in horses (Equus caballus). Journal of Anatomy 206: 557-574. 
Robert, C., Audigié, F., Valette, J., Pourcelot, P. and Denoix, J.M., 2001a. Effects of treadmill speed on the mechanics of the back in the trotting saddlehorse. Equine Veterinary Journal 33: 154-159.

Robert, C., Valette, J., Degueurce, C. and Denoix, J., 1999. Correlation between surface electromyography and kinematics of the hindlimb of horses at trot on a treadmill. Cells Tissues Organs 165: 113-122.

Robert, C., Valette, J. and Denoix, J., 2000. The effects of treadmill inclination and speed on the activity of two hindlimb muscles in the trotting horse. Equine Veterinary Journal 32: 312-317.

Robert, C., Valette, J. and Denoix, J.M., 2001b. The effects of treadmill inclination and speed on the activity of three trunk muscles in the trotting horse. Equine Veterinary Journal 33: 466-472.

Robert, C., Valette, J.P., Pourcelot, P., Audigie, F. and Denoix, J.M., 2002. Effects of trotting speed on muscle activity and kinematics in saddlehorses. Equine Veterinary Journal 34: 295-301.

Roy, S.H., De Luca, G., Cheng, M.S., Johansson, A., Gilmore, L.D. and De Luca, C.J., 2007. Electro-mechanical stability of surface EMG sensors. Medical \& Biological Engineering \& Computing 45: 447-457.

Schuurman, S.O., Kersten, W. and Weijs, W.A., 2003. The equine hind limb is actively stabilized during standing. Journal of Anatomy 202: 355-362.

Sousa, A.S. and Tavares, J.M.R., 2012. Surface electromyographic amplitude normalization methods: a review. Electromyography: new developments, procedures and applications.

St. George, L., Hobbs, S.J., Richards, J., Sinclair, J., Holt, D. and Roy, S., 2018. The effect of cut-off frequency when high-pass filtering equine sEMG signals during locomotion. Journal of Electromyography and Kinesiology 43: 28-40.

Sullivan, G.M. and Feinn, R., 2012. Using effect size-or why the P value is not enough. Journal of Graduate Medical Education 4: 279-282.

Tokuriki, M. and Aoki, O., 1995. Electromyographic activity of the hindlimb muscles during the walk, trot and canter. Equine Veterinary Journal 27: 152-155.

Valentin, S. and Zsoldos, R.R., 2016. Surface electromyography in animal biomechanics: a systematic review. Journal of Electromyography and Kinesiology 28: 167-183.

Van Boxtel, A., 2001. Optimal signal bandwidth for the recording of surface EMG activity of facial, jaw, oral, and neck muscles. Psychophysiology 38: 22-34.

Van Boxtel, A., Boelhouwer, A. and Bos, A., 1998. Optimal EMG signal bandwidth and interelectrode distance for the recording of acoustic, electrocutaneous, and photic blink reflexes. Psychophysiology 35: 690-697.

Williams, J., Gundry, P., Richards, J. and Protheroe, L., 2013. A preliminary evaluation of surface electromyography as a tool to measure muscle fatigue in the National Hunt racehorse. The Veterinary Nurse 4: 566-572.

Winter, D., Rau, G., Kadefors, R., Broman, H. and De Luca, C., 1980. Units, Terms and Standards in the Reporting of EMG Research. A Report by the Ad Hoc Committee of the International Society of Electrophysiology and Kinesiology, Department of Medical Research. Rehabilitation Institute of Montreal, Montreal.

Yang, J.F. and Winter, D., 1984. Electromyographic amplitude normalization methods: improving their sensitivity as diagnostic tools in gait analysis. Archives of Physical Medicine and Rehabilitation 65: 517-521.

Zaneb, H., Kaufmann, V., Stanek, C., Peham, C. and Licka, T.F., 2009. Quantitative differences in activities of back and pelvic limb muscles during walking and trotting 
between chronically lame and nonlame horses. American Journal of Veterinary Research 70: 1129-1134.

Zsoldos, R., Kotschwar, A., Kotschwar, A., Groesel, M., Licka, T. and Peham, C., 2010a. Electromyography activity of the equine splenius muscle and neck kinematics during walk and trot on the treadmill. Equine Veterinary Journal 42: 455-461.

Zsoldos, R., Kotschwar, A., Kotschwar, A., Rodriguez, C., Peham, C. and Licka, T., 2010b. Activity of the equine rectus abdominis and oblique external abdominal muscles measured by surface EMG during walk and trot on the treadmill. Equine Veterinary Journal 42: 523-529. 